\section{$\S 16$. Excitation of Geodesic Acoustic Mode in Toroidal Plasmas}

Itoh, K.

Hallatschek, K. (IPP, Germany)

Itoh, S.-I. (RIAM, K yushu U niv.)

The instability of the geodesic acoustic mode (GAM) in tokamak turbulence is analyzed. It can be caused by dynamic shearing of the ambient turbulence by GAMs combined with the poloidal inhomogeneity of the turbulent flux. The GAMs are more unstable for high safety factors.

The new mechanism of GAM excitation results from the modulation of the flux by the dynamic shearing of the turbulent waves. This dynamic shearing mechanism has been found by numerical simulations in [1]. The analytic theory is derived as follows.

We are interested in the case that the background drift wave fluctuations are in the strong turbulence regime. In such a case, the modification of the drift wave turbulence by the superposed $\widetilde{V}_{E \times B}$ can be evaluated by use of the conservation of wave action. In order to apply this method, we calculate the change of $k_{r}$ of the drift waves. In toroidal plasmas at the outboard midplane, the phase of the dominant modes varies slowly in the direction of the major radius but rapidly in vertical direction, which is a consequence of the ballooning effect. A superposed sheared poloidal rotation shifts the radial wavenumber $k_{r}$ of the drift wave fluctuations with time. (See Fig.1) Let $\theta_{1}$ denote the angle between the mid plane and the wave front. In the absence of GAM, one has $\theta_{1} \simeq 0$ and the phase front starts to rotate, after the zonal flow has been superposed, with the angular frequency $\mathrm{d} \theta_{1} / \mathrm{d} t=\partial \widetilde{V}_{E \times B} / \partial r$. That is, the quantity $k_{r}^{2}$ evolves as $\mathrm{d} k_{r}^{2} / \mathrm{d} t=2 k_{r} \mathrm{~d} k_{r} / \mathrm{d} t=-\left(2 k_{\theta}^{2} s \theta\right) \mathrm{d} \theta_{1} / \mathrm{d} t$ up to the first order in $\theta_{1}$. The perturbation of $k_{r}^{2}, \delta\left(k_{r}^{2}\right)$, ensues as $\delta\left(k_{r}^{2}\right)=-2 k_{\theta}^{2} s \theta \int^{t} \mathrm{~d} t \frac{\partial}{\partial r} \widetilde{V}_{E \times B}$. The drift wave action $\left(1+k_{\perp}^{2} \rho_{\mathrm{S}}^{2}\right)^{2}\left|\phi_{\text {micro }}^{2}\right|$ is adiabatically conserved during a change of perpendicular wavenumber. The change of the turbulence amplitude, $\delta\left|\phi_{\text {micro }}^{2}\right|$, can be computed to be

$$
\delta\left|\phi_{\text {micro }}^{2}\right| \simeq-2 \frac{\rho_{\mathrm{s}}^{2} \delta\left(k_{r}^{2}\right)}{\left(1+k_{\perp 0}^{2} \rho_{\mathrm{s}}^{2}\right)}\left|\phi_{\text {micro }}^{2}\right|
$$

The modulation of the source $F \equiv(S-\nabla \cdot \Gamma) / n_{0}$ is calculated and the amplitude of the $\sin \theta$ component is calculated by the $\sin \theta$-weighted flux surface average as

$$
\widetilde{F}_{1}(r, t)=u_{\mathrm{WH}}\left(\int^{t} \mathrm{~d} t \frac{\partial^{2}}{\partial r^{2}} \widetilde{V}_{E \times B}\right)
$$

with $u_{\mathrm{WH}}=\frac{3 s k_{\theta}^{2} \rho_{\mathrm{s}}^{2}}{\left(1+k_{\perp 0}^{2} \rho_{\mathrm{s}}^{2}\right)} F_{0} L$. The growth rate of the

GAMs oscillation is given as

$$
\operatorname{Im}(\omega)=\frac{q^{2}}{1+2 q^{2}} \frac{R u_{\mathrm{WH}} K_{r}^{2}}{2}-\frac{\mu_{\|} K_{r}^{2}}{2\left(1+2 q^{2}\right)} .
$$

If the condition

$$
q^{2} R u_{\mathrm{WH}}>\mu_{\|}
$$

holds, the drive by dynamic shearing exceeds the stabilization by turbulence viscosity, and the GAM is expected to be excited. An order estimate of the divergence of the particle flux results in $F_{0} \simeq D L^{-2}$, where $D$ is the anomalous particle diffusion coefficient. One has $u_{\mathrm{WH}} \simeq s k_{\perp 0}^{2} \rho_{\mathrm{S}}^{2} D L^{-1}$ for $k_{\perp 0}^{2} \rho_{\mathrm{s}}^{2}<1$. One has the condition for GAM instability by this mechanism as

$$
\frac{s q^{2} R}{L} k_{\perp 0}^{2} \rho_{\mathrm{s}}^{2}>\frac{\mu_{\|}}{D}
$$

Noting that $\mu_{\|} / D$ is close to unity, the GAM is induced by turbulence for the higher $q$ cases that satisfy $q^{2}>q_{\mathrm{c}}^{2} \sim L R^{-1} k_{\perp 0}^{-2} \rho_{\mathrm{s}}^{-2} s^{-1}$. If one considers the application to ion-temperature-gradient (ITG) driven turbulence, the microfluctuations are excited in the range of $k_{\perp 0}^{2} \rho_{\mathrm{s}}^{2} \sim 0.1$ for $s \sim 1$. The GAM excitation is expected to occur when $q^{2}>q_{\mathrm{c}}^{2} \sim 10 \frac{L}{R}$ holds.
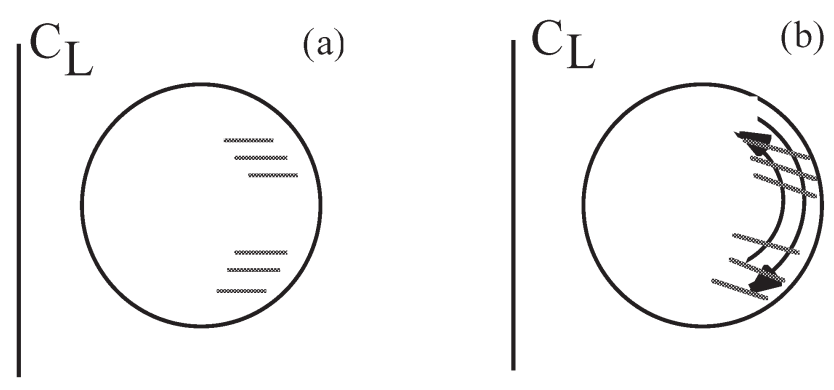

Fig.1 Schematic illustration of dynamic shearing of ambient fluctuations by GAM. Contours of phases of toroidal fluctuations are shown by thick lines in (a). In the presence of poloidally-symmetric shearing motion, the phase contours are modified with up-down asymmetry.

\section{Reference}

[1] K. Hallatschek and D. Biskamp: Phys. Rev. Lett. 86 (2001) 1223

[2] K. Itoh, K. Hallatschek, S.-I. Itoh: Plasma Phys. Control. Fusion, 47 (2005) 451 